\title{
The Future of Social is Personal: The Potential of the Personal Data Store
}

\author{
Max Van Kleek and Kieron O’Hara
}

\begin{abstract}
This chapter argues that technical architectures that facilitate the longitudinal, decentralised and individual-centric personal collection and curation of data will be an important, but partial, response to the pressing problem of the autonomy of the data subject, and the asymmetry of power between the subject and large scale service providers/data consumers. Towards framing the scope and role of such Personal Data Stores (PDSes), the legalistic notion of personal data is examined, and it is argued that a more inclusive, intuitive notion expresses more accurately what individuals require in order to preserve their autonomy in a data-driven world of large aggregators. Six challenges towards realising the PDS vision are set out: the requirement to store data for long periods; the difficulties of managing data for individuals; the need to reconsider the regulatory basis for third-party access to data; the need to comply with international data handling standards; the need to integrate privacy-enhancing technologies; and the need to future-proof data gathering against the evolution of social norms. The open experimental PDS platform INDX is introduced and described, as a means of beginning to address at least some of these six challenges.
\end{abstract}

\section{Introduction}

A key characteristic common to the various kinds of "social intelligence" described in this volume is one of enhanced autonomy through technological support. Such

Max Van Kleek

University of Southampton, University Road, Southampton, SO17 1BJ, e-mail: emaxeecs . soton.ac.uk

Kieron O'Hara

University of Southampton, University Road, Southampton, SO17 1BJ, e-mail: kmo@ecs. soton.ac.uk 
autonomy allows constituents of a society to form new connections with others dynamically as needed, promoting a more adaptive, flexible and robust social fabric than those of traditional structures, in which efficiency leads a majority to rely on a handful of central, fixed intermediaries. This observation immediately prompts the question of whose interests that "efficiency" is designed to benefit — the intermediaries' or the users'.

While we see technology being applied in many contexts to generalise the benefits and enhance the autonomy thus described, the storage of personal information is one area where it has, thus far, been used to power a perverse reversal towards more centralisation. Currently, a handful of dominant platform vendors and application service providers are grappling for control over individuals' personal information, trying to accumulate as many users as possible in order to maximise understanding of every nook and corner of social interaction - a relentless process satirised in Dave Eggers' 2013 novel The Circle, about a company with the totalising slogan "All That Happens Must Be Known". This centralising trend, backed by a surveillance-and-analytics business model, began with the rise of so-called "Web 2.0 ", in which sites became sophisticated apps and content-management platforms designed to facilitate the creation and sharing of user-generated data and content. That content began as a few social network profiles and blog posts, but gradually grew to encompass the entirety of personal data people keep or generate, from files and documents to film and music archives. Thus began a migration of personal digital artefacts from individually-administered personal computers into various information spaces of the web. The assimilation of personal data from personal digital devices has accelerated as Web application and service providers have started to create deep integrations with personal computing devices such as Facebook Home ${ }^{1}$, Windows Skydrive ${ }^{2}$ and Apple's iCloud ${ }^{3}$. Such services have extended the reach of Web services into the intimate digital spaces of one's personal devices, offering backup and management services for these private data collections as well.

What are the implications of this centralisation? Although the ultimate, longterm implications of this shift are not yet fully understood, several immediate consequences are apparent. Fundamentally, the delegation of responsibility for management of one's personal information to third party service providers necessitates relinquishing control over various aspects of how these data are handled and processed, ranging from how they are stored and represented, to how (and when) they can be accessed, as well as to whom access is granted. When third party delegation accidentally-on-purpose serves the increasingly pervasive business model of deriving revenue directly from these data themselves (through targeted advertising or licensing to third parties), platforms are essentially incentivised to collect from as many individuals as possible, and to create an experience or mechanism that further retains them as long as possible to do as wide a range of things as possible. They are also incentivised to disguise the extent of this delegation, for example by embedding

\footnotetext{
${ }^{1}$ Facebook Home - www. facebook. com/home

${ }^{2}$ Skydrive - www. microsoft. com/skydrive

3 iCloud - www. apple.com/icloud
} 
control protocols into complex and legalistic privacy policies whose acceptance is virtually costless (clicking the 'accept' button), binary (yes/no forever) and unconditional, and which are subject to arbitrary change without notice. Platforms get users to disclose as much of their information as possible (to the platforms' benefits) by artificially forcing a tradeoff between participation and privacy; in order to enjoy the most basic features of the Web, users have to give their data away, thereby sacrificing control over their data and potentially their privacy.

This misalignment of incentives between what users want to do with their data and what platform providers want to do with their data has the potential to interfere destructively with the development of context-sensitive applications that promise more effective, personalised, behaviourally-adaptive interactions that rely on richer and more sensitive data models, due to either actual or perceived privacy risks entailed. Moreover, the dependency relationships that result from this process place unprecedented power in the hands of these companies, leaving individuals effectively locked in, and unable to switch to alternative providers without greater effort than it is reasonable to expect a privacy-aware non-technical consumer to devote to the problem; the result of this is an overall reduction of autonomy and mobility, potentially ultimately leading to increased fragility, fragmented data spaces and lost or forgotten data $[74,48]$.

While this business model has thus far been hugely successful at creating extremely profitable services from the likes of Facebook, Twitter and Google, the result has been an increasingly fragile ecosystem in which a majority of Web users have come to rely on an oligarchy of service platforms which are in turn amassing a disproportionate quantity of users' personal information. This centralisation, and accompanying power asymmetry, has occurred not just for Web users from the United States, where most of these services are based, but internationally as well, raising concerns pertaining to each country's sovereign rights of access to data of its own versus other nations' citizens, which have been magnified by the information-gathering practices of the US National Security Agency and others revealed by Edward Snowden in 2013. Indeed, thorny issues pertaining to compliance and enforcement of data protection laws across international boundaries $[6,13]$ represent a serious potential risk for this business model, even as the European Union debates a revision to its pre-Web Data Protection Directive. The EU's weak and unsatisfactory 'safe harbor' rule, which allows data sharing with the United States, conveniently diverting attention away from the unsolved problem of differing approaches to privacy and data, looks especially vulnerable - yet where would the cloud be without safe harbor?

However, a basic assumption that powers these dependence relations and underpins the oligarchy is the disparity between the data management capabilities held by the end-users of the Web from those that provide the hosting and storage. In this chapter, we question this "thin client" model of Web computing by examining an alternative approach that places the responsibility of data management back with the users who own it, but in a way that is natural and manageable, while supporting the same social, dynamic interaction flows they are used to on the Web. This set of capabilities we refer to as personal data stores (PDSs), the technical goal of 
which is to augment user computing devices with secure data storage, hosting, and sharing capabilities which can be used to archive and manage valuable information longitudinally, as they interact with one another and third parties respectively.

Our aim in this chapter is to derive the requirements for personal needs for such a platform through insights from the field of Personal Information Management (PIM). To begin with, it is worth reviewing in more detail the dilemmas and asymmetries that current management of "big data" has created, across the public and private sectors, and why the individual is understandably at a loss. Although PDSs cannot conceivably solve or even address all these issues, we should keep them in mind in order to understand the extent to which it makes sense to include PDSs as part of a more equitable longer-term settlement. Second, we present a brief summary of existing platforms being used to manage personal information and their characteristics. The chapter concludes with a discussion of how these platforms may change the socio-economic landscape of the Web, and the ways personal data is shared, collected and handled.

\section{The Dilemmas of the Data Economy}

Although we would not hazard a guess as to who originated the phrase, we do know that data has been called "the new oil" on many occasions. Of course, the image is intended less as an indication of the deep issues at the core of the data economy, and more as a neat way of conveying excitement in a Powerpoint bullet. However, it is indicative, because it can be taken in various, not necessarily exclusive, ways. Oil is a source of great wealth. It is a key factor in many other production and transport processes. It is an essential lubricant. It needs to be mined (well, drilled to be precise) to produce value. It brings great riches to the small number of corporations big enough to exploit it. It raises exchange rates and therefore prices to the detriment of other industries. It has been known to impoverish those whose property is drilled, as elites cream off the main wealth with the help of rapacious corporations and corrupt government. It has, on occasion, led to revolution and the overthrow of anciens regimes.

Presumably not all these phenomena associated with the old variety are intended to be predicated of the "new oil." Yet we, as data subjects, presumably want to be sure that we get the good things and not the bad. It is anyway a misleading comparison, because data has properties that oil does not. Data is about people, and can be compromising. Data is generated by people, not by aeons-old trees and animals which have no issues of privacy or dignity. It is not a dwindling resource - we are far fromapproaching the time of "peak data." It is not a rival good if I enjoy its use, that does not preclude your exploiting it at the same time. Data becomes valuable when aggregated across communities. Data is a covert way of financing content and services; if the service you receive is free, then you must be the product. 
Data is connected to us by an umbilical relation - we generate it in all sorts of ways, and it is about us. We create and provide it; we leave trails of it; it is inferred about us. Yet the flip side of this is that it expresses things we find important (indeed, Luciano Floridi [26] argues that our data are an inalienable part of our identity). By providing a route for others to understand what we are, or what we have done, or where we are situated, it can threaten our privacy, or our dignity, or our autonomy, by diluting the privileged first-person access to our own experience. It creates the possibility of our being counted, measured, judged, steered or influenced without our knowledge by mysterious forces or organisations who may or may not have our best interests at heart. And if the data about us don't exist, we can be profiled, and treated as a standard member of a small demographic, whether this is accurate or not. Maybe this means we get more interesting advertisements - or maybe we will be treated as a potential terrorist and denied access to an aeroplane without adequate explanation.

Because of this, data is regulated. Data protection law is intended to strike a balance between the public good (which may include commercial benefits) of data use, reuse and sharing, and the private good of privacy and individual control. Data subjects have certain rights over their personal data - but not the rights of ownership. If I browse an online bookstore, then I have thereby created a load of data which is of value to someone else. They have constructed a website, and therefore claim ownership rights of the trail - the data results from their investment. In case of dispute, they will cite my consent to their use of my data via some privacy policy that I probably never noticed. It may be argued that I benefit from the collection of this data, because it gives the bookstore sufficient evidence to suggest other books to me that may interest me (and we know, from Amazon's early experience, that a good recommendation algorithm will easily outperform a human recommender). If I were given ownership of my browsing data, then there would be no incentive for the online bookstore to collect it, so it wouldn't be collected, and no-one would benefit from it. If data subjects owned their personal data, then third parties wouldn't bother to collect it, and the data economy would remain a glint in Google's eye.

Data protection is not there to protect privacy; that is at best its secondary purpose. But worse, data protection was a concept developed for the world of standalone databases, not the connected, networked Web with which we are familiar. A tangled skein of legislation struggles to cope with the realities of the personal data economy. Trading personal data goes on at scales previously unimaginable. A user goes online, and literally dozens of organisations will be tracking his or her behaviour. There is talk that this will benefit the data subject, via better devices, better websites and better recommendations; the main 'benefit', arguably, is to become a better target for marketing. I sacrifice my privacy and aspects of my intimate identity for a better class of spam.

Some economists [56] have argued that the release of personal data is a good thing for wider society, as it reduces information asymmetries and enables capital and currency to be allocated in a more informed way. Hmmm, maybe in an ideal world. But arguably the data economy is functioning by ramping up the asymmetries - data-using organisations not only know much more about the use they are making 
of their data than I do, they now in many cases know more about me than I do. I, the poor data subject, am sitting at the bottom of so many data asymmetries that the idea that I am too informed for the public good is surely laughable.

Furthermore the concepts of data protection, so valid and timely when they were first introduced, seem at best quaint in 2014. Data minimisation is a great principle, but is it realistic in a world where five billion Google searches and half a billion tweets are generated every day, not to mention the colossal number of mobile phone locations that are logged? Can the use limitation principle be of value in a world where serendipitous reuse is the order of the day? In the data economy, after all, the primary use of data $i s$ its secondary use. Do notice and informed consent have any meaning in such a world? Do we want to be notified of everything, when our lives are becoming increasingly complex and the choices we already have to make are multiplying? What, when I click the 'yes' button, am I consenting to? Doesn't virtually everybody treat the 'yes' button as an opening to a new and exciting online experience, rather than a notification of the commencement of a complex business relationship which entails a certain amount of risk for the insouciant clicker? Can this truly be glossed as informed? One might as well say that the fox is giving informed consent to the hunt when he tunnels under its boundary fence in search of prey.

But how to react to this? We must surely admit the many benefits that data can bring to the subject. Understanding oneself is an important part of managing one's health or consumption. The benefits accrue not only to the individual, but also wider society. Effective public health, transport and crime management are facilitated by giant quantities of accurate micro-level data. So data sharing with government and businesses cannot be made too difficult to do.

One potential way forward is to move from the current model of data protection, based on regulating the collection of data, for a defined purpose, centred on the data controller, and governed by the consent of the individual. The 'footprint' of the data now stretches far beyond the immediate context and purpose, and regulating for the moment of collection looks anachronistic. A number of commentators, including advocates of 'big data' [40], argue that the time is ripe for a move from subject consent to user accountability. Such a model would regulate the uses of data, and would be centred on the subject who would be given a greater, and less binary, measure of control. For example, Novotny and Spiekermann argue for a three-tier information market, with key distinctions in terms of responsibilities and liabilities between data subjects, service providers, a second tier service space that provides essential support for the top level service relationship, and a tertiary space in which data from the top level relationship is reused on an open but restricted market [47].

What kind of control should the subject be granted? Ownership brings responsibilities as well as rights, and as we noted may mean that potentially valuable data would never be collected at all. Furthermore, many thinkers are nervous that the concept suggests that people's identities are basically property and commodities, with all the dehumanisation that implies. On the other hand, a human rights approach, for example based on Article 8 of the European Convention, is something of a blunt instrument, and the article is frustratingly vague as to what we actually should do 
in a particular context. Furthermore, Article 8 is in place and agreed across Europe and many other countries, yet our data is still plundered by the data barons.

In the remainder of this chapter, we will attempt to answer some of these very difficult questions, by tracing a particular idea through conceptual beginnings to a concrete architecture. The next section will consider the notion of personal data or personal information in more detail.

\section{Doing Things With Personal Data}

Despite the clear importance of the concept, "personal data" means different things to different disciplines and communities. In this section, we will consider these different views of data with a view to understanding what capabilities it could afford for subjects, given sufficient access and control. We begin by looking at some of the different definitions, then from the perspective of Personal Information Management look at some of the activities around data and data management, and complete the section by considering the technological support for such activities.

\subsection{What Constitutes "Personal Data"? Legal and Operational Definitions}

The standard way to conceive personal data is via its legal definition, based on data protection law. This conception has two advantages: first of all it is widely accepted and understood, and secondly it matches the legal liabilities that any PDS management system will need to confront and accept. Personal data, on this definition, is data relating to an identifiable individual. There are a number of issues and indeterminacies here - identifiable by whom? using what methods? in what context? but these need not detain us here, except to note that they do not make things any easier. The legal definition has not really kept up with technical developments, and it is clear that the ability to identify a data subject is highly context-dependent [44]. 'Personal data' is the usual European term, but in the US it can be known as 'personal information' or personally identifiable information'.

There are strong sanctions against the misuse of personal data without the data subject's consent, but data sharing can still take place if the data controller deidentifies the data by removing identifiers from it or aggregating it (whereupon the new dataset is no longer personal data). There are many techniques for this [77], and there is also a major and unresolved debate [51], [15], [44] about whether de-identified data can be made re-identifiable by cross-referencing it with other datasets, using so-called 'jigsaw identification' methods. For instance, the information that a girl in a dataset is pregnant is not identifying, and therefore not personal data, but combined with the information that Mary Jones is the only girl in the dataset, clearly a possibly unwelcome inference can be drawn about the all-too- 
identifiable Mary Jones. In this chapter, we will not consider the issues raised by such technicalities in detail, except to note that (a) they impinge on data sharing practices and may impose complex liabilities that will be hard to predict, and (b) they can be side-stepped in many cases if the data is exposed by the data subject, who can therefore be assumed to have given consent for use of that personal data by others, given that he or she made the decision to share it in the first place. In the context of giving data subjects greater control over the data that is about them, this is clearly a vital factor to consider.

If we now move from the legal definition, and consider this latter context, an alternative understanding emerges of personal information as the information over which a person has some interest or control, in order to negotiate their environment or order their lives (so, distinct from data in which a person has a commercial interest only). This type of personal information or data is much more in tune with an intuitive understanding of what data means to me. And as one would expect, it would include a great deal of crossover with the legal definition of the data from which I am identifiable, but it is likely to include data of which I am the owner, but from which I could not be identified at all (e.g. photographs I have taken, from which it may even be possible that other people might be identifiable, and hence which might be personal data with respect to those people).

The uses to which such data may be put might be social or entertainment, or could be work-related, consumption-related, or administrative; it might also have no obvious immediate use, but be stored in case it should have value later on. The data may come from several sources: it could be self-generated, deliberately created, a by-product of other kinds of activity, shared with friends or colleagues, open data from the Web, or have been officially bought or licensed from the (legal) owner. Therefore the data in which a person has an interest will almost certainly be of various types of legal status. Personal information in this sense has been investigated by researchers in Personal Information Management (PIM), and we can draw on some of their insights.

The task of identifying all of the kinds of data a person might need to keep, manage and use is a complex and not easily scoped task. Researchers in PIM have derived various working definitions of personal information in order to effectively scope their field of study, and and have made progress towards potential functional classifications for kinds of personal information. One such classification by Jones et al. [34] is visible in Table 1.

Jones takes an approach that distinguishes among different kinds of information by how it relates to the individual in question; whether the individual experienced it, kept it, sent it, or received it, or whether this information refers to the individual or his or her activities. The categories About me and Relevant to me are controversial because these definitions do not require individuals to be aware of the existence of the information; it thus establishes a sphere that goes beyond the scope of information experienced by the user. We discuss the potential implications of including such information within the scope of PDSes in attentional challenges. 


\author{
Category \\ 1. Owned/controlled by me \\ 2. About me \\ 3. Directed towards me \\ 4. Sent (provided) by me \\ 5. Experienced by me \\ 6. Relevant (useful) to me
}

Examples

e.g., Email, files on our computers

e.g., my credit/medical history, web history

e.g., phone calls, drop ins, adverts, popups

e.g., Emails, tweets, published reports

e.g., Pages, papers, articles I've read

e.g., Somewhere "out there" is the perfect vacation, house, job, life-

long mate

Table 1 Categories of Personal Information - Jones's proposed taxonomy of personal information. [34]

\subsection{Activities Around Personal Information}

Each person can access, use and manage information in many different ways in their everyday activities. Moreover, there is considerable variation among the ways that different people manage their information, as documented in studies of people's office and home information environments predating personal computers altogether [38]. As a result, it has been relatively difficult to come up with a single characterisation encompassing all of these activities; several classifications have been proposed. Returning to the PIM literature, Jones et al. propose a categorisation centering about a distinction between finding, keeping, and a set of "M-level activities", which encompasses managing and organising information archives (Table 2) [34]. Whittaker et al's slightly different categorisation, meanwhile, simply identifies 3 classes: keeping, management, and what they call "exploitation", as follows:

\begin{tabular}{|c|c|}
\hline Jones [34], Jones and Teevan [67] & Whittaker et al. [73] \\
\hline $\begin{array}{l}\text { Re-)Finding } \\
\text { Keeping } \\
\text { Meta-level activities (managing, } \\
\text { maintaining) }\end{array}$ & $\begin{array}{l}\text { Keeping } \\
\text { Management } \\
\text { Exploitation }\end{array}$ \\
\hline
\end{tabular}

Table 2 Categories of PIM activities - Table comparing Jones's 3-tiered categorisation of information activities [34] versus that proposed by Whittaker [73].

Jones's classification introduces finding as a primary activity that people perform; his definition spans a set of common behaviours including discovery [17], information foraging [54], orienteering [66, 10], searching [68] among other related behaviours in which people purposefully seek information or serendipitously encounter it in the course of other information activities. Once this information is found, information is either consumed and internalised, or kept in an external archive, or both, and this process of saving information externally is referred to as keeping. Beyond this activity of archiving, individuals might return to their archives to organise, update, or trim them; such activities are referred to as the $\mathrm{M}$-level, for manifold meta- and management, hence $M$-level, activities. Whittaker then includes 
a fourth category of behaviours, exploitation, referring to the the set of ways in which the information is used and applied.

Among such uses, while the foremost might be to inform an individual making a decision, many other uses of information also exist. For example, information might be created for the explicit purpose of reminding a person of past or future events, activities or details. Other purposes might be to measure and keep track of the timeevolution of some phenomenon so that it can be easily understood. When this measurement is about the individual's own activities, the purpose might be for providing feedback, which may be vital for behavioural modification domains such as cognitive behavioural therapy (CBT)-like programmes. This feedback may, in turn, along with other information, collectively serve to motivate further activity or behaviour. Finally, information may serve the purpose of external cognition, in which information is created or manipulated for the purpose of facilitating understanding or problem solving. This set of activities is often referred to as sensemaking [55].

\subsection{Supporting Information Activities}

Technological support for each of these information activities has demonstrated the potential to change not only how they are conducted, but the contexts in which they are applied. One salient example is that of Web search engines, originally created for Web page information retrieval, but which have become a nearly ubiquitous tool for accomplishing tasks across a much broader variety of activities, spanning both desktop and mobile. Another area is in supporting longitudinal curation; tools that automatically perform off-site, incremental, and continuous backup such as Apple's Time Machine ${ }^{4}$ have become commonplace, allowing end-users to make their stored data more resilient to accidental deletion or data loss.

Yet technological support for most of the other aforementioned personal information activities, including reminding, sensemaking, discovery and orienteering, has remained rudimentary. Reminding in PIM tools, for example, has until only recently been limited to clock/calendar-based alarms that need to be explicitly set for a specific date and time, despite the rich variety of "off-line" strategies people have naturally adopted for their own uses [9]. While the basic calendar alarm remains heavily used, its precision, brittleness and intrusiveness have been documented to undermine effectiveness, sometimes through extended "snooze wars", in which users repeatedly dismiss alarms, resulting in their piling up over time. The alarm can end up a burdensome annoyance, instead of providing the intended assistance.

The mismatch between people's data management requirements and the technology to support it is not, of course, restricted to PIM. As another example where the promise has not been borne out, Privacy Enhancing Technologies (PETs) [72] have yet to make a mark either. They too have failed to transcend the perennial problem

${ }^{4}$ Time Machine - www . apple.com/uk/support/timemachine/ 
of demanding an investment of time and resources that few want to make, or want to have to make. They also put a relatively inflexible barrier between individuals and organisations, while the individual may in fact have very context-dependent requirements (it is handy, for example, for an online fashion company to know my size, even if I do not want this parameter value bruited abroad). Takeup has been predictably anaemic.

\section{Personal Data Stores}

Yet surely technology must be part of the solution to a technologically-driven problem. Technology creates data, with the connivance of the data subject, and tools have emerged for large-scale players to exploit their vast datastores. The concept we wish to explore in this paper, in response to the foregoing discussion of the challenges and context, is that of the Personal Data Store (PDS). This is a locus of control which leaves open a number of the key questions about ownership and property, while giving power to data subjects. Our aim in this chapter is to set out some of the possibilities of PDSs, and to try to show that at least some of the above dilemmas can be addressed with them. Clearly PDSs will not be the full story - but they should be part of the solution. We hope to suggest some ways this could happen, and how indeed it has happened, and to illuminate the potential by refining our account to produce a specific example of a PDS architecture.

The aim of PDSes is to start to narrow the aforementioned data inequality by bolstering the capabilities of individuals for managing, curating, sharing and using data themselves and for their own benefit. The idea is not for such capabilities to replace services, nor for individuals to take their data out of the rich ecosystems that exist today (a feat which would be practically impossible, not to mention potentially destructive), but instead to enable people to collect, maintain and effectively derive value from their own data collections directly on the device(s) under their control. The combination of such capabilities and derived value provides an incentive for individuals to take responsibility for, and invest effort in, the preservation and curation of their data collections, turning to external third parties for specialised services only where needed. The aim of such development would be to try to restore some balance by providing a locus for subject-centric management of data, to complement (and in some cases replace) the current paradigm of organisation-centric data management.

Arriving at an operational definition, we define PDSes as follows:

A personal data store is a set of capabilities built into a software platform or service that allows an individual to manage and maintain his or her digital information, artefacts and assets, longitudinally and self-sufficiently, so it may be used practically when and where it can for the individual's benefit as perceived by the individual, and shared with others directly, without relying on external third parties.

This description leaves undefined the kinds of activities that might constitute "managing", "maintaining", "controlling fully" or "using" this information, nor 
even what kind(s) of information, owned by whom, that we are talking about. Fortunately, significant insight pertaining to many ways individuals readily use information (in both on-line and off-line contexts) has been gained through studies conducted at the intersection of psychology and computer science, particularly the Human-Computer Interaction (HCI) research community. Beyond insights about existing information practices, various ideas have been proposed dating back nearly a century about how technology might change human-information and human-human relationship, modulated by new emerging information technology.

\subsection{Historical Reflections From Memex ...}

The genesis of an individual-centric personal data archive pre-dates digital computers entirely, to Vannevar Bush's Memex vision of 1945 [12], which proposed a mechanical framework for supporting the collection, archiving, and organisation of information to facilitate later cross-reference and retrieval. Among the important contributions of this article was the significant emphasis on reducing the effort needed to capture and retrieve information, due effort being the primary impediment towards effective and frequent information use. To this end, Memex proposed that individuals could wear capture devices on their bodies (a camera strapped to the forehead), store such information compactly, conveniently and indefinitely, and retrieve it later through an associative mechanism modelled upon the human memory, queried naturally via gesture.

Two additional early projects that explored how such information archives might be realised were Ted Nelson's Xanadu [45] and Douglas Engelbart's NLS [22]. Both proposed that information environments could be interlinked through a global network of knowledge sharing, demonstrating many ideas in the 1960's that would not be realised in commercial systems for decades. While the former focused on hypertext and distributed collaboration, the latter focused on structured data collections, including data navigation, creation and management. Engelbart demonstrated an actual prototype of NLS in 1969, capable of synchronous collaboration, complete through a graphical user interface, that incorporated dynamic hierarchies, hyperlinks, and multi-view representations

The introduction of the personal computer (PC) in 1984 provoked the development of the first generation of digital personal information management tools, consisting of a variety of application software products designed to help individuals create and maintain collections of digital data, ranging from flexible, schemaagnostic personal database systems like Filemaker ${ }^{5}$, to specific data types, such as digital calendaring tools, and "digital Rolodex" address books. Seeking to appeal to the first generation of personal computer users, many of these applications borrowed metaphors from paper-based information collection tools, from the notion of "documents", to that of files and folders, and even notebook ledgers and personal diaries.

\footnotetext{
${ }^{5}$ Filemaker - www. filemaker. com
} 
Along with this deliberate shaping of digital information into forms designed to be familiar with paper information organiser came interaction metaphors and organisation methods for them; from deletion of information by "throwing in the rubbish bin" to "desktop" and "filing cabinet"-based based information organisation and arrangement.

Meanwhile, research in personal information management continued to pursue the vision put forth by Memex, towards methods of automatically building archives of personal life activities and experiences, so that these might be used as external memory prostheses. The pursuit of this vision was partially responsible for the development of handheld and early wearable computing technology, such as the Xerox PARC Tab [61], arguably the first hand-held computer, which ran arguably the first automatic location-based personal lifelog, PEPYS [46]. Many systems that captured other aspects of context and activities soon followed, such as the Remembrance Agent by Rhodes et al., and the life archive by Clarkson et al., both at the MIT Media Lab's "Cyborg" Wearable Computing group. Since the breadth of kinds of activities and experiences that such systems captured transcended paper documents, such research required re-thinking the shape of data away from paper-metaphors to other kinds of collections, including information streams (e.g., Lifestreams [25]) and chronological lifelogs, such as MyLifeBits [28].

The third, and potentially most profound, transformation of digital information tools occurred with Web 2.0, the rise of a "social Web" replete with dedicated apps and services for managing and sharing nearly any kind of previously imagined personal information, ranging from the sensitive and intimate to the public.

Meanwhile, the data proliferated too. Seeking to monetise the flood of information people were putting online, markets for personal information quickly began to emerge, prompting concerns over privacy, security, and rights of access, which in turn have driven government and regulators' interest towards giving citizens more protection over various aspects of how data about them could be collected and handled. This led to international efforts to craft data protection legislation, as discussed above. In terms of the provision of data to individuals, such legislation so far has focused on allowing data subjects to inspect the data an organisation holds about them; on receiving a subject access request, the organisation is obliged to correct inaccuracies, and to respect requirements that the data is not used in any way which may cause damage or distress, and that the data is not used for direct marketing purposes.

However, this is a fairly minimal power which is hardly congruent with the increasing clamour concerning rights to data, including the spread of enforced transparency of data from the private sector [27] and the vogue for freedom of public sector information [50], and technology (and technology policy) together with new attitudes to transparency bring more possibilities. In the UK, a government initiative called midata [63] is working to bring about the logical next step of customers getting direct and unfettered access to data kept about them by companies (other similar initiatives include the US Blue Button initiative ${ }^{6}$ and the French Mesinfos group ${ }^{7}$ ).

6 www4.va.gov/bluebutton/.

7 mesinfos.fing.org/. 
The ultimate success of midata will be contingent on several important steps in both technology and regulation, most particularly including realising effective tools such as personal data stores for letting individual users easily consume, consolidate and make use of this data once it is made available.

\section{2 ... To Mydex: Birth of the PDS concept}

Independent of such legislative approaches, both academic and industry-led efforts also began to commit resources to research towards identifying ways that end-user citizens might, in the face of the vast growing repositories of data being held about them, enjoy more control and privacy. An academic consortium known as Vendor Relationship Management (VRM) at Harvard's Berkman Center was realised to conduct multifaceted research into socio-legal-econo-technical approaches that might be employed. Among the products of this research was a vision that users might stand as their own information brokers, and start to act as peers with service providers, capable of negotiating fair and equitable mutual terms of data use during interactions with them [2]. Out of this work emerged the earliest mentions of Personal Data Stores for realising such capabilities in the context of online e-commerce, inspiring more than a dozen different Personal Data Store offerings, platforms and services backed by commercial start-ups since 2001 [1].

As an example, consider Mydex, whose proof-of-concept offering dates back to 2009 [32]. Mydex designers worked with data-handling organisations to develop systems to support data transfer and sharing governed by consent and identity verification. Design principles included putting the individual PDS owner in sole charge of consent giving and revocation with a simple 'on/off' switch; giving the individual sole access to the private encryption key; verification of all organisations wishing access to data; and comprehensive data sharing agreements going beyond Data Protection Act protections. The business model for Mydex is still experimental, but currently the idea is to fund the stores by charging organisations for access to data; if the charge is set low enough, then they should save by side-stepping other access costs (e.g. the costs of writing a letter to the data subject). The Mydex services are currently free of charge to the individual. Mydex exploits cloud infrastructure with open source software, but its PDSs are discrete collections of files encrypted and controlled by the individual, including - and this seems prescient after the Snowden revelations ${ }^{8}$ - the ability to choose the location of the data centre in which the PDS is stored. Similar open source personal data storage containers include The

8 www.theguardian.com/world/the-nsa-files, www.ub.uio.no/fag/ informatikk-matematikk/informatikk/faglig/bibliografier/no21984. html. 
Locker Project ${ }^{9}$, data.fm ${ }^{10}$, Owncloud ${ }^{11}$, and OpenStack ${ }^{12}$, each of which provides various degrees of easy-to-set-up 'personal cloud' software that can be used to store and host content on the user's own server on the Web.

A consistent theme of commentary in this area has seen Personal Data Stores (PDS) as important, if not essential, capability for end-users towards growing a healthier global "personal data ecosystem". For example, an independent study commissoned by The World Economic Forum documented ways that the value of personal data might be further "unlocked", citing Personal Data Stores as a core enabling mechanism to turn end-users from consumers into more autonomous data brokers [11]. A separate comprehensive analysis by Ctrl-Shift on emerging commercial PDS platforms and offerings projected an enormous economic opportunity for PDS services in the next five years [1]. In their view, PDSs are the key to making sense of the myriad data sources that now surround us, from data we volunteer, to the data that commemorates observations of our behaviour, to the data inferred about us, combined with the data we generate via management of our personal affairs (e.g. in health or finance), and also bringing in data about our activities as customers or consumers, including our contributions to loyalty card schemes.

\subsection{Failure to Launch: Barriers to PDS Adoption}

Yet despite the extensive needs analysis and market potential identified, early personal data store offerings have thus far failed to attract substantial attention from users. While a number of factors are likely responsible, so the lack of interest among users has been attributed to the fact that many of initial PDS platforms have sought to simply re-create existing end-user experiences offered by popular apps and Web platforms, rather than creating new functionality. Despite the benefit that these PDS offerings provide in terms of data security, users are often less compelled to try something new if the tangible experience nothing new, while data security remains an abstract, inestimable threat which does not necessarily easily compel behaviour change [5]. Finally, since the very purpose of PDS offerings is to protect user data from third party access, these platforms cannot derive revenue from user data and must resort to subscription models - always less attractive to new users than than offerings that are completely free to use.

On top of these suppressors of the positive impulse to manage data, we must also remember that the markets work pretty well for some (the most powerful) operators, and so there is a great deal of inertia around. A dogmatic view of revealed preferences of course suggests that individuals' lack of interest in the technology shows they have no desire to curate their own data. They happily click on privacy policies

\footnotetext{
9 lockerproject.org.

10 data.fm.

11 owncloud.org.

12 www. openstack.org.
} 
they have never read, and they buy the goods that are marketed to them, at least in sufficient quantities to justify the marketers' costs. 'Push' models seem to be in the ascendant, because the data oligarchs are the only agents with access to the bigger picture of what data is held about you, what can be inferred from that data, what services are available, and how you relate to the general data context. 'Pull' models struggle, because individuals cannot see the opportunities that are around. In short, the argument is often made that the technological direction of travel is more or less set, that it serves the public good, that the public is uninterested in any alternative, and so, to coin a phrase, "get over it." This deterministic model has been called Zuckerbollocks [49], and it is important to challenge and resist it.

Heath et al. write [32] that "there is market evidence that [the person-centric model of control over personal data] is starting to establish itself," but even they see a challenge to getting the model to work. Three conditions need to obtain simultaneously, on the account of Heath et al: PDSs must (i) make life simpler/better for the individual, (ii) appeal to data consumers by solving some of their problems (e.g. costs, or legal liability), and (iii) solve some pressing challenge that is holding back developers and entrepreneurs in this space. To these three, we can add a fourth, which is to rejig current data protection thinking. At the moment (2014), there are three key roles in the standard model of data protection: the data subject, the data controller and the data processor. The owner of a PDS is none of these (or none exclusively - he or she is likely to be all three at various times), and it is hard to see how individuals can exercise autonomous control over the data that affects them without some recognition of them as active agents in a different kind of role. Furthermore, data protection legislation is intended to cover cases of personal data being misused by others; it does not cover cases where individuals accidentally (or deliberately) identify themselves. Of course, this is a reasonable starting point for protection, but if it is the only principle, it means that if an individual 'takes charge' of his or her data, he or she loses the cover of Data Protection Acts.

\section{Six Not So Easy Pieces: Challenges towards Realising the PDS Vision}

The goal of providing individuals with the capacity to maintain their own information longitudinally imposes a number of challenges to supporting the kinds of information activities we have described. In particular, we see six broad categories of challenge to be met; the first, most fundamental of which pertains to effective longitudinal keeping. Enabling individuals to keep their data safely for a long time, while ensuring its continued accessibility and usefulness impacts both the data formats and methods used to store them. For example, since a person's physical computational hardware is likely to fail with age, methods need to be in place for ensuring robustness to such failures, such as multi-device replication and easy migration from older to new devices over time. Moreover, as evidenced by Moore's law [59], since the technical capabilities and properties of such data storage devices and platforms 
are likely to change fundamentally, PDSes must be designed to accommodate (and take advantage of) such changes as they arise. The devices and technologies that have made the PDS vision possible date back only a couple of decades, whereas a safe haven for data such as we are envisaging might well have to last a working lifetime (before we even consider the issues surrounding inheritance of data after a death).

A second challenge is allowing individuals who might have little or no experience in the intricacies of data management to cope with the burden of data security and longitudinal maintenance. Using current tools and services, for example, managing your data yourself still means taking pains to ensure that one's personal data is not lost to hardware and software failure, malicious attacks, or safely migrated to new platforms and devices; such efforts require vast investments of time, effort and expertise. A general lack of expertise or willingness to do this means that people currently rarely know how, or bother, to back up or consolidate their data. Thus it is no surprise that individuals have been motivated to outsource maintenance of their data to third parties, such as cloud providers. In order to facilitate autonomy from such services, therefore, PDSes must seek to support directly, and automate where possible, tedious data maintenance tasks that have plagued PC users for decades. Such automation could both ensure compliance for promoting data security and integrity, such as continuous backup regimes, thereby countering recent studies of the extremely low compliance of personal data backup and security maintenance practices [16, 29].

A separate set of challenges arises from the shift back from service-provider controlled data storage to a user-centered model of data management. Although this will re-empower users to control the organisation of their data spaces, and eliminate the pervasive problem of data fragmentation [35], [31], the challenge with the increased flexibility that this approach affords is that it requires re-consideration of how third-party applications and services can interact with such data, which have traditionally been pre-defined to operate on a fixed, typically application-provider established, set of data representation(s) and manipulations. In a consolidated, usercentric data model, on the other hand, such representations may be be specified or modified by the individual, or by some other third-party application(s) on behalf of them, and thus applications themselves must be designed to accommodate such variability among representations.

The need to comply with local, national and international data handling requirements pose a fourth set of challenges. In particular, if PDSes are to support the storage of identifiable information, or more critically, regulated sensitive information such as individuals' medical records, then PDSes must implement a variety of security standards (e.g. [41]) to ensure secured storage. Perhaps more difficult might be achieving compliance with the additional data handling requirements imposed by these regulations beyond how it is stored and encrypted; in particular, key handling requirements and guaranteeing aspects of physical access to the machine(s). The integrity of data must also be secured - for instance, although a patient should have the right to challenge and correct inaccurate medical data, if the PDS is to store a version of medical data that is likely to be used (for example, in support of medical 
treatment in a foreign country), the data would need not only to be accurate, but also of appropriate provenance in order to be properly adapted to the standard workflows of medical treatment.

Even if PDSes were to achieve all of the aforementioned goals, individuals would still face the fact that service providers would inevitably continue to profile and amass information about them, as long as it aligned with their incentives to do so (and it is hard to imagine that it will not - for instance, a service provider may need to gather a large amount of personal data in order to ensure correct and appropriate billing for its services). Thus, if PDSes are to give users the degree of autonomy and independence from profiling, they would need to include privacy-enhancing technologies, such as IP anonymisers, user-agent randomisation and cookie blocking. This may be difficult or impossible to do on "closed" platforms such as iOS that prevent these techniques because they are perceived as "hacking".

Perhaps the ultimate set of challenges, however, pertain to accommodating change as it affects both the information itself and the practices and activities surrounding it, over the years that a PDSes is intended to operate. Technologies that bring in new ways that data is used and generated seem to be introduced every quarter, placing new demands how this information needs to be accessed, created and used. The most recent examples include wearable computing and "always on" wearable sensor technology, from simple devices such as Fitbits ${ }^{13}$ and Fuelbands ${ }^{14}$ that unobtrusively but nearly constantly measure simple aspects of an individual's activity, to complex computational devices that can both deliver and capture information in high fidelity and quantity anywhere, such as Google Glass ${ }^{15}$. Such devices, as well as innovative new apps, can, in some cases, bring about changes in norms pertaining to people's activities, including the ways people think about technologies themselves.

Looking forward at some of the ways such technologies might impact information activities, some have looked at the possible consequences and implications that ever-increasing information capture and access might have on the kinds of activities mentioned above. While Bell and Gemmel have argued [8] that such increased capture and access could create near-perfect records of our daily lives, allowing people to examine with unprecedented scrutiny their everyday activities, others such as Mayer-Schonberger have argued that such a utopian views overlooks a great number of potential unintended consequences [40].

The difficulties that this community has encountered have led us to reconsider, from the ground up, the need(s) these platforms are meant to address, so that they can be used to design a platform that will fulfill needs beyond secure data storage, towards new applications that promote the more effective use of data in both personal and social contexts.

\footnotetext{
${ }^{13}$ Fitbits - www. fitbit.com

${ }^{14}$ Nike+ Fuelband - www.nike.com/fuelband

${ }^{15}$ Google Glass - www. google.com/glass
} 


\section{Survey of Online Data Platforms and Services}

Given this characterisation of the various kinds of personal data and activities around it, we can identify the ways that current online services fulfil the needs towards people's information types and activities.

Table 3 lists the top five personal data cloud platforms by number of users. While Facebook may not be considered an end-user personal data storage provider of the likes of Dropbox, it remains one of the world's largest brokers of personal information. Of particular interest is the introduction of its Timeline feature in December 2011, which took the format of a visual chronological lifelog starting at the individual's birth. In order to compel users to backfill information about their lives into their Timelines from before they joined Facebook, the platform introduced prompting questions, asking for information such as all of the places one has lived, one's family members and favourite activities. Somewhat surprisingly, the negative "backlash" surrounding Timeline has been predominantly surrounding its aesthetic and usability [52], rather than its privacy intrusiveness, except among a small but vocal minority [19]. This aggressive strategy, however, has succesfully driven millions of individuals to divulge rich histories of their lives with unprecedented fidelity.

Facebook only supports the storage of very specific information forms, spanning status updates, likes, photos, messages to individuals and so forth. Among the remaining services, Google Drive, Dropbox and Skydrive support general file storage, with the former two providing full versioning history support, while Skydrive providing versioning only for MS Office documents with a full paid Skydrive Pro membership ${ }^{16}$. iCloud, meanwhile, in a move congruent to their push for their mobile devices to render user-visible filesystems obsolete', does not support the general storage of files.

A survey of why people used the file-oriented storage services revealed that while backup had previously been the main reason for using online cloud services, multidevice access and sharing/collaboration have quickly eclipsed backup for reasons people use such services online [70]. The primary use of Facebook, meanwhile is to stay connected with others, as well as several emotional reasons, spanning reasons of self-actualisation and to fulfill the need to belong [43].

However, these services primarily pertain to the management of a fraction of the personal data encompassed by Jones's definition above, specifically "data owned/controlled by me". If we also extend consideration to online services that host and collect "data about me" as well, there are now an increasing number of sensor-driven apps and services that facilitate the tracking of various, routine aspects of everyday life activities, spanning purchases, movements, wellbeing vital statistics; we list such life tracking sites in Table 4.

While both categories of services broker significant amounts of data, these do not generally meet the requirements for personal data stores, as service providers

\footnotetext{
16 Skydrive Pro- http://office.microsoft.com/en-001/

office365-sharepoint-online-enterprise-help/

manage-document-versions-in-skydrive-pro-HA103158256.aspx
} 

Facebook Profile incl. Timeline; Friends; Events; Group memberships; Biographical history; States favourites; Preferences; Message archives; Liked pages, im- ages, products; Places visited.
Google Drive Any files; Google Docs; calendar; G+ profile; identify and profiles of friends; search history; page access history; bookmarks; locations visited
iCloud iWork Documents, Photos, Calendars, Passwords (Keychain)
Dropbox Any files
Skydrive Office Documents; Any files.

Table 3 Most popular commercial cloud data storage providers - Most popular service-centric data storage providers in 2014, listed with descriptions of kinds of user data managed.

\begin{tabular}{|c|c|c|}
\hline Service & Description & Logging Method \\
\hline Foursquare; FB Places & Visits made to points of interest & Manual check-ins \\
\hline Moves & $\begin{array}{l}\text { Complete history of a person's move- } \\
\text { ments throughout the day as recorded } \\
\text { from smartphone app }\end{array}$ & Sensed via mobile \\
\hline Mint; BUDGT & $\begin{array}{l}\text { Access to personal banking records } \\
\text { (tracking spending) }\end{array}$ & Sensed \\
\hline Withings & Weight, blood pressure, & Sensed \\
\hline $\begin{array}{l}\text { Garmin HRM; Polar } \\
\text { HRM; Cardiio App }\end{array}$ & $r$ Heart rate over time & Sensed \\
\hline Zensorium Tinke & Blood volume pulse, stress markers & $\begin{array}{l}\text { Manual } \\
\text { ment }\end{array}$ \\
\hline $\begin{array}{l}\text { Fitbit; Fuelband; Jaw- } \\
\text { bone }\end{array}$ & - Daily activity levels & Sensed \\
\hline Wattvision; Stepgreen & Energy consumption & Sensed \\
\hline $\begin{array}{l}\text { Moodpanda; Mappiness; } \\
\text { Gotafeeling }\end{array}$ & ; Mood/emotion/stress & Experience Sampled \\
\hline $\begin{array}{l}\text { CalorieCounter; Foodu- } \\
\text { cate }\end{array}$ & - Daily calorie consumption & Manual \\
\hline
\end{tabular}

Table 4 Activity and life trackers - Popular web "lifelogging" services that facilitate the capture and logging of everyday life experiences.

ultimately remain in control how this data is stored, secured, and have full access to its contents. Other services, meanwhile have been launched with their primary offering centered on end-user privacy and control; such services (examples of which are listed in Table ??) are sometimes referred to as the first generation of "personal data store" offerings.

Personal.com Cloud svc for keeping important structured data of specific schema types (passwords, contact details)

Mydex Cloud sve centered around specific structured data and identity verification

Table 5 Early PDS Offerings - Personal Data Store offerings which encrypt data to provide a high degree of user data security, e.g., only the user has access.

These offerings protect user data through encryption and by adhering to data handling standards; however, user data still physically reside in data centres operated by 
these service providers, where they ultimately remain under their control. Similarly, these services thus far are both highly restrictive on the kinds of information they are designed to manage, with support for a handful of different information types in specific schemas.

A different approach that embraces a "DIY" model [57] for PDSes are software packages that people can install on their hardware devices of choice, in order to provide data management and security capabilities. An example of such software packages are Table 6. While aerofs and bittorrent sync are proprietary commercial software packages, the remaining are released under libre, open source licenses [71].

The open source model provides a number of advantages in terms of realising PDSes. First, these licenses allow these systems to be appropriated, in whole or in parts, and mixed with other systems, in order to construct bespoke PDS functionalities in any ways seen fit. It also encourages transparency by allowing anyone (and everyone) to consult, verify and improve its code, while at the same time making it difficult to hide malicious code within them, such a malware.

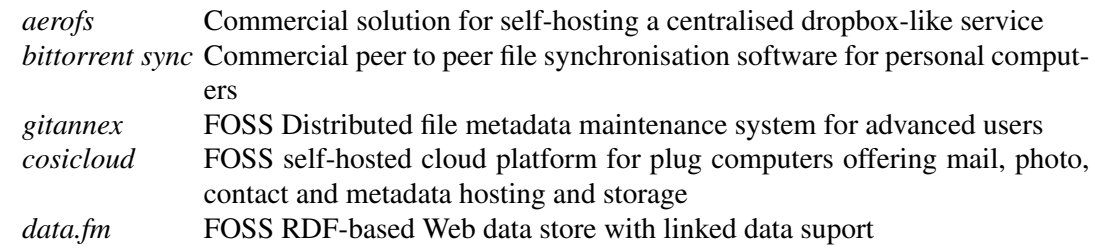

Table 6 Self hosted data management software - A sampling of commercial and FOSS software designed to facilitate management of personal information.

It is worth noting that while these DIY PDS platforms are largely platform and hardware agnostic, there are a number of hardware personal data archiving solutions for personal use, ranging from simple external hard drives, automatic-backup solutions that provide version histories, such as Apple's Time Capsule ${ }^{17}$, NAS storage devices (e.g. WD MyClouds ${ }^{18}$ ), to systems that provide data resilience, access control and some degree of data security such as Drobo ${ }^{19}$.

In terms of support for the kinds of aforementioned PIM activities, these examples demonstrate that the majority of personal data services have thus far focused on prioritising data durability and multi-device data access. Beyond data backup, a few provide full data versioning, and some offer data security guarantees as well.

Sharing is another kind of support that is central to all of these services, reflecting their common roots in social "Web 2.0" services. This comes in terms of real-time collaboration for some (e.g.,, Google Drive and Skydrive), while nearly all of the cloud and DIY platforms above provide some support for asynchronous collabo-

\footnotetext{
${ }^{17}$ Apple Airport Time Capsule - www . apple.com/airport-time-capsule/

${ }^{18}$ WD MyCloud www.wdc.com/en/products/network/networkstorage/

${ }^{19}$ Drobo - www. drobo. com
} 
ration, including disconnected operation (e.g., Dropbox Google Drive, Skydrive, gitannex).

The specific approaches taken to supporting PIM activities also vary considerably; some have bundled application front-ends, or sets of application utilities"baked" into them (such as Google Drive and WD MyCloud), while others simply function as generic storage containers for existing applications (e.g., Dropbox and gitannex). Still others stand as their own platforms for future PIM apps and services (e.g. cosicloud). Two trends are clear, however; first, that the commercial centralised' cloud offerings currently outpace the self-hosted options in terms of features and lowest immediate visible cost, both in terms of subscription costs (due to the pervasive freemium models) and in terms of time and effort to set up and use. In terms of long-term costs, however, the advantages of provider-hosting are less clear; the DIY approaches promise much greater flexibility by facilitating the creation of re-appropriable, custom-tailorable PIM solutions. Moreover, since selfhosted solutions place the ultimate responsibility on the user for the maintenance of his or her data, they provide much greater potential for long term data durability and security.

Based on this perceived disparity between cloud-hosted and DIY solutions, we organised an open source community effort around identifying and realising advanced PIM support in self-hosted, DIY platforms. The goal of this effort was to identify how to realise a system that would overcome the barriers to using selfhosted platforms while leveraging its benefits; specifically supporting research questions on how to better support users' long-term data retention and management needs.

\section{INDX: A Research Programme Around Personal Data Stores}

The substantial challenges just described towards realising an actual PDS platform that achieves the goal set out in the introduction makes deriving a requirements specification daunting. Such a specification would require a well-defined and limited set of capabilities, provided in sufficient detail to be realised in a software (or software-hardware system). Yet, it is not clear how such a set of capabilities (out of many) should be chosen, nor how to choose a such a set to satisfy the requirement of minimality (to avoid overspecification). Nor, finally, is it entirely clear how to verify whether any such set could reach its intended goal.

Therefore we believe a research-centric, rather than development-driven, approach may be the most suitable for bridging the gap between the high-level challenges discussed and the evaluation of potential solutions. Towards this end, we have begun a research project centred about a set of core questions for investigation, and an open experimental research PDS platform called INDX ${ }^{20}$.

${ }^{20}$ INDX source code and distributions - http://indx.es 
The purpose of INDX and the research efforts around it, are several; from a research coordination perspective, it aims to serve as a common ground where various research communities may identify interrelated issues. This is a particularly critical role, as the kinds of work emerging from usable security, privacy, data durability, decentralised social systems, could both be informed by, and used to inform others about how approaches might fit into an integrated picture of future information management systems.

The second role is to serve as a base platform upon which various PDS technical and interface experiments can be tested in a real world setting. To this end, INDX will provide a basic implementation of what one might consider the most elementary kinds of services that PDSes are likely to need. We outline the specific such functionality in the next section. The reason that a complete, open implementation of a basic set of components is necessary for evaluation is to provide essential functionality to enable PDS researchers to focus on particular problems one at a time, rather than having to re-implement these basic components per experiment.

The third, and perhaps most critical reason for INDX is that a concrete implementation is necessary to even start to interrogate many of the goals pertaining to how the systems might be used by indivdiuals. A deployable implementation of a PDS architecture opens up the possibility of running field experiments, which can be vital to understanding how individuals might perceive or adopt functionality in actual use. Just as the social mechanisms of the Web could not be effectively studied until years after it was built (and continues to evolve), the various interface and interaction mechanisms of PDSes may set off different usage(s) that would altogether be difficult to anticipate prior to deployment. Such is particularly important for personal information management practices, which have been shown to be highly slippery and idiosyncratic; people appropriate and change the ways they use the tools in their collections in unexpected, creative ways in order to satisfy their particular needs.

\subsection{Base functionality of the INDX PDS Platform}

The base architecture of INDX consists of three components; a versioned database for semi-structured data, a distributed identity subsystem, and management logic that glues the components together. Each is described below, along with rationale for its design.

\subsubsection{The Data Store}

A key question in implementing the core component of a PDS is choosing the "right" database - what kind of data model should it use? What query language should it support? How should it store the data to ensure longevity? 
As databases have evolved over the years, many kinds of database models have been proposed and improved. The INDX design process brought us to consider many popular database types, including "traditional" relational databases, document oriented (or "NoSQL") databases, graph based data stores, "XML" databases, and RDF triple stores, to name a few. Each offers a few distinct advantages over the others, and many open source implementations exist of each type.

Since there are several advantages to using pre-existing databases, the most obvious of which is the fact that using mature, open-source software is likely to be more reliable and require less engineering than creating a bespoke solution from the ground up. Beyond this purely practical development consideration, there is a greater argument for being database-agnostic [18], rather than sticking to a single implementation. In order to realise the PDS vision of longevity, an unavoidable fact is that hardware and software is going to change dramatically, as will the database systems built on top of them; moreover, there may be a need to accommodate a variety of different data demands, with uses and needs continually increasing, as data streams become more numerous, personal data archives archives become larger, and query and sharing functionality is tasked with increasingly challenging applications. What may make sense to run on a single "conventional" PC today might need to be run on a thousand nodes in some virtualised computer architecture in the future in order to accommodate an individual's increased storage and query capacity.

Therefore, using the age-old engineering principle of modularity, we sought to create the INDX PDS as an adapter on top of one or two basic underlying database systems. This decision has enabled us to target multiple databases at the outset, ranging from desktops and servers to mobile devices.

The question of finding an appropriate data and query model for PDSes is a more delicate question because the design choices made at this level are visible to, and thus directly affect, application developers, and to a certain extent, end-users. A variety of considerations need to be made when selecting the data model; first, whatever target model is chosen must be sufficiently flexible to accommodate (with reasonable transformation) the kinds of data that the platform will be managing. A poorly suited data model for the target will likely introduce inefficiencies that will either slow down performance, increase complexity or both.

Fortunately, most of the aforementioned data models are fairly general, each with specific characteristics; for example, relational databases require data to be factored into tables, which assumes a certain degree of data regularity; XML databases represent data as hierarchical structured documents; more general document-oriented stores manage collections of (either structured or unstructured) documents with limited metadata (comprising sets of keys for retrieval), while RDF ultimately represents data their granular components: triples.

Another dimension is that certain types of databases more typically afford guarantees that others do not; for example, many relational databases offer grades of ACID (Atomicity, Consistency, Isolation and Durability) guarantees [42], while few document-oriented or RDF triple stores do, partly due to technicalities arising from realising these guarantees in these settings. An additional advantage to relational databases is that extensive research on them has yielded well-known methods to 
"tune" performance, such as ways to factor tables to avoid otherwise computationally expensive query operations, the creation of indexes and so on, whereas such methods and query performance predictability is remains less well established for other database types.

The culmination of these observations, with the availability of an highly respected implementation have led us to target a relational database, Postgres [64], for desktop and server hosted INDX stores.

\subsubsection{Datastore Management}

However, despite its large feature set, Postgres does not, "out of the box" meet all of the capabilities required of a PDS by the definition we arrived upon earlier. Given the need for PDSes to continue to meet changing information needs over an individual's lifetime, it is rather unlikely that any database will ever be devised at any point in time that will be able to fulfill all future information needs itself. Thus, this is where the design of the PDS has to provide incremental functionality extension, again, through encapsulation and modular design.

One of the immediate such functionality that must be added in order to use Postgres as the core data store is support for schemaless storage. Being a relational database, this is not straightforward; typical scenarios of the deployment of Postgres involves having a database programmer specifically create a bespoke set of schemas per data type being stored, consisting of tables and related views. Yet, in terms of PDSes, such needs may not be known at the time of set-up, and may change dramatically over time; moreover, it is practically impossible to know at design time the structure of all the data any user might want to store.

A second example also relevant to long-term data retention was providing the capability of a revisitable history of all data objects kept in the store. There are many uses for such a history, such as letting a user retrieve old versions of their objects, such as their documents, that were subsequently lost or altered, or determining how particular objects were changed over time. Such capabilities have started to become available in commodity software such as Apple's Time Machine, platforms such as Dropbox and Skydrive, as well as many collaborative software tools. Thus, we believe that it such a capability will soon become a standard capability assumed by users.

Other capabilities that in the works for INDX include managing replicated copies (for enhanced resilience against datastore failure and corruption), sharing (such as object-level sharing support), and encryption for handling sensitive data. Such platform-level data capability allows PDS platform application writers to take advantage of sophisticated functionality and data security without having to implement them within apps themselves, allowing the unilateral improvement of data handling without adding application-wise complexity.

An important piece of functionality that the PDS management logic also has to assume is to access control, which involves orchestration of at least three separate components: access control policies specified by the user and stored as rules, the 
database's own gatekeeping mechanisms for granting access to the data kept within, and digital identities of users and applications requesting access, described next.

\subsubsection{Distributed Identity Management}

The current predominant model of identity management is that service providers perform this management directly for users; for example, service providers allow users to create principals with them, and provide authentication mechanisms as well. This model is inconvenient for a decentralised model of interaction, however, as it requires users to register a new principals with every single individual's PDS prior to interacting with them.

Distributed identity management protocols [36] offer a solution to this problem, by separating the problem of identity establishment and verification from its use. This permits, for example, an individual to grant access to sensitive data in their PDS to a verifiable identity of an entity, for example, their GP, even if ther GP has never previously interacted with their PDS. Currently popular distributed identity management implementations include OpenID [58], WebID [33], Mozilla Persona [75].

A related problem that is distinct to identity management is that of allowing third parties to request and securely receive access to data (with the user's permission). For this purpose, protocols such as OAuth [30] and SAML [4] have been developed and implemented across a large number of data providers, including Facebook, Instagram, and others. Such mechanisms allow these particular parties to continue to share data on behalf of the user once permission has been granted once, without subsequent user intervention.

INDX's reference implementation uses OAuth in conjunction with OpenID to allow interoperability with current Web services, particularly for the purpose of permitting transparent archiving of content that users distribute across the Web. It currently supports the archiving of content posted to social networking sites and services such as Twitter and Facebook, activity logging sites such as Nike+, Withings, and Moves, financial tracking sites such as Mint, open data sources such as OpenWeatherAPI, with support for other services to follow.

\section{Looking Forward: Functionality for Future Information Management}

In this final section, we wish to touch upon a few potential ways that PDSes might change the ways individuals will work with information in the future. A key goal will be to achieve consolidated data models from heterogeneous sources, for which we discuss the role of semantic technology and ontology matching and alignment algorithms; and the implications . 


\subsection{The Challenge of Automatic Consolidation}

If one were to make an assumption that Personal Data Stores will eventually be able to draw in information obtained from hundreds to even thousands of third party data sources, for example, ranging from social networking posts to retail sites to Wikipedia to one's electronic medical record providers, so that such data may be safely archived, versioned and conveniently accessed, a question remains - how will this information be organised?

While this information could be kept separate and archived in its original form as provided, there are significant advantages to a user if this heterogeneous data is consolidated. By consolidation, we imply the act of combining complementary information from multiple sources into fewer, coherent and more complete and consistent representations. If this is done, like information items can be displayed in a consistent fashion, making coherent presentation and manipulation of items simpler; such consolidated information can be used by the user (and by the user's applications) uniformly, effectively eliminating the aforementioned problems of fragmentation mentioned earlier. The advantages to the user of a single consolidated data model are many, and we discuss a few of the potential ways this may enable applications to do more sophisticated things for users later in this section.

If all information service providers adopted the a single unified schema for all information coming into and out of them, this goal could be achieved relatively simply, since data records from separate sources could be directly compared. However, it is fairly well accepted that achieving such a singular data representation is as unlikely as convincing the entire world to speak exactly one dialect of a single language; the degree of diversity and continued independent evolution of systems practically guarantees that this will never happen [7].

Thus to tackle this challenge, we must perform a kind of information integration, in which data are transformed into a consistent representation. For any pair of fixed sources, bespoke mapping could be specified by a programmer manually. However, if the applications are not known, or if the data came in arbitrary forms unknown in advance (such as if they came directly from a user), other methods must be employed. It is this latter situation that is likely to be quite common for PDSes, particularly considering the wide range of potential data and applications a user might need. We briefly discuss how semantic technology and ontology matching algorithms may be able to help.

\subsubsection{Semantic technology}

Research pertaining to the Semantic Web has looked at methods by which automatic inference over heterogeneous information can be made possible by grounding such representations in ontologies related through ontology languages, such as OWL [3]. Such semantics establish a framework by which machine translation of information representations become made possible through the formal stated connections made about such representations. The role of semantic reasoners thus are to take informa- 
tion represented in such formats, along with their source ontologies, and to allow relationships among such information items to be deduced.

A requirement for such technology to work, however, is that all information providers provide appropriate mappings for their information representations against common ontologies using languages such as OWL. Thus far, few Web data sources outside of research and a few specific domains have embraced such techniques, making the use of such ontology languages, meaning that other approachese may also have to be employed. One such is the use of automatic ontology matching algorithms.

\subsubsection{Ontology matching: automatic and interactive methods}

Two other approaches have been taken to this problem; one is the use of machinelearning techniques for ontology matching (e.g. [24, 21], or instance matching [65, 14]). In such approaches, an algorithm is given a collection of examples of ontologies (or instances) and their corresponding semantic relationships, and the algorithm extrapolates properties to new, yet unseen relationships. This remains a rather computationally difficult task, however, and these methods have remained highly imperfect.

One promising approach has been to use such methods in combination with interactive approaches, that is to let users help such matching algorithms out when they get stuck. The end-user programming community has sought interfaces that can leverage information from non-expert individuals, who are empowered to assist and orchestrates the process of reconciliation at various levels of specificity. Systems that use this approach include "mash-up makers" (such as Mashmaker, [23], Marmite [37], Vegemite [76]) and interactive data workbenches, such as DataPalette [69].

\subsection{Defragmentation and "Placeless" Data}

One of the greatest advantages of the Web is that it has started enable pervasive information access; for an increasing proportion of the world's population, people can now access any information, anytime, anywhere from their desktops or mobile devices in nearly any setting [53]. Yet, the silos on the web have created artificial "places" in themselves; so now it is necessary "go to facebook" or "log into my university's portal" or "go to my health care provider", using the dedicated search and navigation facilities of these sites in order to get behind their walled gardens even when the information being sought is the user's own information!

Such walls impede individuals' abilities to quickly access information needed, and in some cases, entirely preclude the ability for this information to be effectively cross-referenced, by preventing links from being established between these data items and increasing the barrier to accessing them. The result is often that the 
user experience of the Web has reverted back from the Memex vision of being able to navigate fluid "association lines" of investigation aimed to complement the associative mechanisms of human memory and creative thinking, instead getting back to a series of online disparate bulletin board systems.

The vision of the PDS may reverse this at least for one's personal information, by providing consolidated representations of all of the information items distributed across silos that can be arbitrarily cross-referenced and linked. Doing this has its subtleties, however; as argued by, Marshall et al, "simply archiving" by harvesting a person's out of all of one's third party services necessarily decontextualises from the context of its original location, application or Web service in which it was created or found [39]. In order to avoid having this loss of context, PDSes could provide "wormholes" from the consolidated representation - which is better suited for sensemaking, to the individual serv ices hosting the rich context of content.

\subsection{Supporting information management for life: Context-sensitive automation and behaviour change}

Since it can be easily argued that the most valuable features of tools are the ones that are the greatest felt, we briefly touch on a few ways that the capabilities afforded by PDSes might directly impact people's lives.

The all too familiar feeling of data loss that occurs when we have had a hard drive fail, or the frustration that arises from not being able to find a particular important document or photo demonstrates the potential for technology to save people from distress in many immediate and direct ways. The position of personal information tools, as the most intimate and direct mechanism for satisfying a majority of our information needs, means that small changes in these tools can have substantial long-term effects.

Across many of the biggest information management problems are a host of wellknown techniques that are simply not used because they are simply too time consuming, require expertise, or that people simply forget. For example, data loss can be practically avoided in relatively simple ways through the creation of off-site backups and vigilance in continuing to back data up over time. However, the low-compliance rate to backup regimens simply comes from the fact that people are often either too busy, forgetful, or simply do not know how to carry out such backups regularly. Similarly, limited time, attention, effort and expertise serve as the root cause for many other problems concerning long-term data preservation and access, including disorganisation, ensuring data security, and accidental deletion.

One potential solution to all such problems is the judicious application of automation in supporting a broader set of information management and maintenance practices. Just as spam filters transparently and automatically remove unwanted mail to save people from having to delete it themselves, or Apple's Time Machine continuously creates generational backups of the information on one's desktop and notebook without the user usually even being aware of it, we can imagine other in- 
formation management activities being facilitated by more of such "attention free" support.

A particular kind of automation that has thus far been technically challenging to realise but well-suited to the capabilities afforded by PDSes is context-aware and adaptive automation that is sensitive to a user's needs, location, and activities. Since PDSes consolidate multiple information streams about a person's sensed activities (such as through wearable activity sensors or apps), it can consolidate the most complete digital "shadow" of the individual. This shadow, can, in turn be used by applications to provide attention-free automation support; for example, by using information about one credit card statement (such as from Mint) with one's current location (sensed via one's smartphone) and purchase history (collected in one's PDS over a long term), a future application might infer automatically that one is at risk of going over credit limit and intervene, either by warning the user, or automatically transfer money on his or her behalf to avoid over-transaction fees. Many such context aware scenarios have been proposed before (e.g. [62], [60], [20]), but their inability to get accurate, high-dimensional data of the user's context have impeded progress. PDSes seem an appropriate solution to this, particularly in situations such as the above where the involved in the inference is highly sensitive and personal, such as one's bank account balance, current location, medical conditions, and so on.

When such context-sensitive and adaptive approaches are applied to health and wellbeing, it can be used to play a role delivering better personalised coaching and intervention support. Simple forms of fitness coaching are already becoming available on the market, usually delivered as part of low-cost commercial activity sensors such as Nike's FuelBand, or Withing's body scale and blood pressure products. However, few of these applications are able to perform sophisticated tailoring due to the limited information available about the user from these single, simple sensor streams. Therefore, the kinds of multi-stream consolidation of user context may be helpful here towards more effective digital support in wellbeing maintenance, intervention and recovery.

\section{Conclusion}

In this chapter we have attempted to position the notion of Personal Data Stores as a (partial) response to the pressing problem of the autonomy of the data subject, and the asymmetry of power between the subject and large-scale service providers and data consumers. Given what Novotny and Spiekermann have called the "missing governance of personal data markets" [47] threatens to undermine subject trust in data sharing practice, and given that data sharing underlies not only a series of very valuable public services but also a whole economy, PDSs are highly suggestive of a means of putting the data subject at the centre of the data market's institutional structure.

The notion of 'personal data' is, for obvious reasons, in thrall to a legal definition that governs liability and policy, but the narrow legalistic coverage that this subtends 
should surely be supplemented by a more intuitive notion of the data which is of interest, importance or value to individuals. Such a rethink would help both individuals, many of whom are concerned, if only in the abstract, that their privacy is being undermined by the collection, storage, aggregation and mining of their data, and data consuming organisations, many of which are concerned about a potential backlash. The rules governing ownership of data seem unlikely to change, as this would hamper the development of an equitable data economy, but regulatory and technical models are emerging in which the rights and responsibilities of various stakeholders are redistributed. PDSs are part of that emerging picture. It is also worth pointing out, however, that even with an unchanged regulatory position, PDSs have made some progress (e.g. [32]) — and the regulatory position is unlikely to remain unchanged in the charged atmosphere (at the time of writing) caused by the Snowden revelations and the revisions to the EU's Data Protection Directive.

Earlier, we set out six challenges facing PDSs, and described a reference implementation called INDX. The intention for INDX was not to make a claim that it would in its current state (or ever) solve all of the challenges, but to serve as a common artefact around collaborative research discourse for investigating sociotechnical issues and user needs. As a functional open platform, our hope is that it might be adopted as an instrument that accelerates research towards more flexible and adaptive information environments that assume dramatically different forms and shapes than our current models of silo-encapsulated hegemonies in the cloud.

\section{Acknowledgements}

The work described in this chapter is supported through an interdisciplinary ESPRC Research Grant EP/J017728/1, The Theory and Practice of Social Machines. The design and development of the INDX platform has benefitted from contributions from SOCIAM researchers, including Daniel Alexander Smith, Laura Dragăn, Markus Lucsak-Roesch, Ramine Tinati, and Dave Murray-Rust, and with direction and advising from Sir Nigel Shadbolt, Sir Tim Berners-Lee, and Dame Wendy Hall.

\section{References}

1. The new personal data landscape. Technical report, 2011.

2. J. M. Agustin and W. M. Albritton. Vendor relationship management. 2001.

3. G. Antoniou and F. Van Harmelen. Web Ontology Language: OWL. In Handbook on ontologies, pages 67-92. Springer, 2004.

4. A. Armando, R. Carbone, L. Compagna, J. Cuellar, and L. Tobarra. Formal analysis of SAML 2.0 web browser single sign-on: breaking the SAML-based single sign-on for Google Apps. In Proceedings of the 6th ACM workshop on Formal methods in security engineering, pages 1-10. ACM, 2008.

5. A. Bandura. Self-efficacy: toward a unifying theory of behavioral change. Psychological review, 84(2):191, 1977. 
6. D. Banisar and S. Davies. Global trends in privacy protection: An international survey of privacy, data protection, and surveillance laws and developments. John Marshall Journal of Computer \& Information Law, 18(1), 1999.

7. L. Bannon and S. Bødker. Constructing common information spaces. In Proceedings of the Fifth European Conference on Computer Supported Cooperative Work, pages 81-96. Springer, 1997.

8. C. G. Bell, J. Gemmell, and C. Rosson. Total recall. Dutton, 2010.

9. V. Bellotti, B. Dalal, N. Good, P. Flynn, D. G. Bobrow, and N. Ducheneaut. What a to-do: studies of task management towards the design of a personal task list manager. In Proceedings of the SIGCHI conference on Human factors in computing systems, pages 735-742. ACM, 2004.

10. D. Benyon and K. Höök. Navigation in information spaces: supporting the individual. In Human-Computer Interaction INTERACT'97, pages 39-46. Springer, 1997.

11. Boston Consulting Group. Unlocking the value of personal data: From collection to usage. Technical report, 2013.

12. V. Bush. As we may think. 1945.

13. L. A. Bygrave. Data protection law. Kluwer Law International, 2002.

14. S. Castano, A. Ferrara, and S. Montanelli. Matching ontologies in open networked systems: Techniques and applications. Journal on Data Semantics V, pages 25-63, 2006.

15. A. Cavoukian and K. El Emam. Dispelling the Myths Surrounding De-identification: Anonymization Remains a Strong Tool for Protecting Privacy. Office of the Privacy and Information Commissioner, Ontario, 2011.

16. A. Chervenak, V. Vellanki, and Z. Kurmas. Protecting file systems: A survey of backup techniques. In Proceedings Joint NASA and IEEE Mass Storage Conference, volume 3, 1998.

17. E. H. Chi, P. Pirolli, K. Chen, and J. Pitkow. Using information scent to model user information needs and actions and the web. In Proceedings of the SIGCHI conference on Human factors in computing systems, pages 490-497. ACM, 2001.

18. N. Chohan, C. Bunch, C. Krintz, and Y. Nomura. Database-agnostic transaction support for cloud infrastructures. In Cloud Computing (CLOUD), 2011 IEEE International Conference on, pages 692-699. IEEE, 2011.

19. S. Choney. Facebook timeline poll: 'overwhelming negative' reaction. Jan 2012.

20. A. K. Dey. Understanding and using context. Personal and ubiquitous computing, 5(1):4-7, 2001.

21. A. Doan, J. Madhavan, R. Dhamankar, P. Domingos, and A. Halevy. Learning to match ontologies on the Semantic Web. VLDB Journal, 12(4):303-319, 2003.

22. D. C. Engelbart and W. K. English. A research center for augmenting human intellect. In Proceedings of the December 9-11, 1968, fall joint computer conference, part I, pages 395410. ACM, 1968.

23. R. Ennals, E. Brewer, M. Garofalakis, M. Shadle, and P. Gandhi. Intel Mash Maker: join the web. SIGMOD Rec., 36(4):27-33, 2007.

24. J. Euzenat. An API for ontology alignment. Proc ISWC '04, pages 698-712, 2004.

25. S. Fertig, E. Freeman, and D. Gelernter. Lifestreams: an alternative to the desktop metaphor. In Conference companion on Human factors in computing systems, pages 410-411. ACM, 1996.

26. L. Floridi. The Ethics of Information. Oxford University Press, 2013.

27. A. Fung, M. Graham, and D. Weil. Full Disclosure: The Perils and Promise of Transparency. Cambridge University Press, 2007.

28. J. Gemmell, G. Bell, R. Lueder, S. Drucker, and C. Wong. Mylifebits: fulfilling the memex vision. In Proceedings of the tenth ACM international conference on Multimedia, pages 235238. ACM, 2002.

29. M. A. Grasso, M. J. Yen, and M. L. Mintz. Survey of handheld computing among medical students. Computer methods and programs in biomedicine, 82(3):196-202, 2006.

30. D. Hardt. The oauth 2.0 authorization framework. 2012.

31. T. Heath and C. Bizer. Linked data: Evolving the web into a global data space. Synthesis lectures on the semantic web: theory and technology, 1(1):1-136, 2011. 
32. W. Heath, D. Alexander, and P. Booth. Digital enlightenment, Mydex, and restoring control over personal data to the individual, pages 253-269. IOS Press, 2013.

33. G. Huang and K. Mak. WeBid: a web-based framework to support early supplier involvement in new product development. Robotics and Computer-Integrated Manufacturing, 16(2):169179, 2000.

34. W. Jones. Keeping Found Things Found: The Study and Practice of Personal Information Management: The Study and Practice of Personal Information Management. Morgan Kaufmann, 2010.

35. D. R. Karger and W. Jones. Data unification in personal information management. Communications of the ACM, 49(1):77-82, 2006.

36. H. Koshutanski, M. Ion, and L. Telesca. Distributed identity management model for digital ecosystems. In Emerging Security Information, Systems, and Technologies, 2007. SecureWare 2007. The International Conference on, pages 132-138. IEEE, 2007.

37. J. Lin, J. Wong, J. Nichols, A. Cypher, and T. A. Lau. End-user programming of mashups with vegemite. In Proc. IUI '09, pages 97-106. ACM, 2009.

38. T. W. Malone. How do people organize their desks?: Implications for the design of office information systems. ACM Transactions on Information Systems (TOIS), 1(1):99-112, 1983.

39. C. C. Marshall. Challenges and opportunities for personal digital archiving. Digital: Personal Collections in the Digital Era, pages 90-114, 2011.

40. V. Mayer-Sch'onberger and K. Cukier. Big Data: A Revolution That Will Transform How We Live, Work and Think. John Murray, 2013.

41. E. McCallister. Guide to protecting the confidentiality of personally identifiable information. DIANE Publishing, 2010.

42. P. Muth and T. C. Rakow. Atomic commitment for integrated database systems. In Data Engineering, 1991. Proceedings. Seventh International Conference on, pages 296-304. IEEE, 1991.

43. A. Nadkarni and S. G. Hofmann. Why do people use facebook? Personality and individual differences, 52(3):243-249, 2012.

44. A. Narayanan and V. Shmatikov. Myths and fallacies of "personally identifying information". Communications of the ACM, 53(6):24-26, 2010.

45. T. H. Nelson. Literary Machines: The Report On, and Of, Project Xanadu Concerning Word Processing, Electronic Publishing, Hypertext, Thinkertoys, Tomorrow's Intellectual Revolution, and Certain Other Topics Including Knowledge, Education and Freedom. Nelson Ted; Schooley's Mountain, NJ: distrib. by Distributors, 1987.

46. W. M. Newman, M. A. Eldridge, and M. G. Lamming. Pepys: Generating autobiographies by automatic tracking. In Proceedings of the Second European Conference on ComputerSupported Cooperative Work ECSCW'91, pages 175-188. Springer, 1991.

47. A. Novotny and S. Spiekermann. Personal information markets and privacy: a new model to solve the controversy, pages 102-120. IOS Press, 2013.

48. W. Odom, R. Banks, D. Kirk, R. Harper, S. Lindley, and A. Sellen. Technology heirlooms?: considerations for passing down and inheriting digital materials. In Proceedings of the SIGCHI Conference on Human Factors in Computing Systems, pages 337-346. ACM, 2012.

49. K. O'Hara. Are we getting privacy the wrong way round? IEEE Internet Computing, 17(4):89-92, 2014.

50. K. O'Hara. The information spring. IEEE Internet Computing, 18(2), 2014.

51. P. Ohm. Broken promises of privacy: responding to the surprising failure of anonymization. UCLA Law Review, 57:1701-1777, 2010.

52. T. Olmstead. Facebook timeline and users: Not quite a love affair. Jan 2012.

53. M. Perry, K. O'hara, A. Sellen, B. Brown, and R. Harper. Dealing with mobility: understanding access anytime, anywhere. ACM Transactions on Computer-Human Interaction (TOCHI), 8(4):323-347, 2001.

54. P. Pirolli and S. Card. Information foraging. Psychological review, 106(4):643, 1999.

55. P. Pirolli and S. Card. The sensemaking process and leverage points for analyst technology as identified through cognitive task analysis. In Proceedings of International Conference on Intelligence Analysis, volume 5, pages 2-4, 2005. 
56. R. A. Posner. The economics of privacy. American Economic Review, 71:405-409, 1981.

57. D. Purdue, J. Dürrschmidt, P. Jowers, and R. O'Doherty. Diy culture and extended milieux: Lets, veggie boxes and festivals. The Sociological Review, 45(4):645-667, 1997.

58. D. Recordon and D. Reed. OpenID 2.0: a platform for user-centric identity management. In Proceedings of the second ACM workshop on Digital identity management, pages 11-16. ACM, 2006

59. R. R. Schaller. Moore's law: past, present and future. Spectrum, IEEE, 34(6):52-59, 1997.

60. B. Schilit, N. Adams, and R. Want. Context-aware computing applications. In Mobile Computing Systems and Applications, 1994. WMCSA 1994. First Workshop on, pages 85-90. IEEE, 1994.

61. B. N. Schilit, N. Adams, R. Gold, M. M. Tso, and R. Want. The parctab mobile computing system. In Workstation Operating Systems, 1993. Proceedings., Fourth Workshop on, pages 34-39. IEEE, 1993.

62. T. Selker and W. Burleson. Context-aware design and interaction in computer systems. IBM systems Journal, 39(3.4):880-891, 2000.

63. N. Shadbolt. Midata: towards a personal information revolution, pages 202-224. IOS Press, 2013.

64. M. Stonebraker and L. A. Rowe. The design of Postgres, volume 15. ACM, 1986.

65. F. Suchanek, S. Abiteboul, and P. Senellart. PARIS: probabilistic alignment of relations, instances, and schema. Proc. VLDB '11, 5(3):157-168, 2011.

66. J. Teevan, C. Alvarado, M. S. Ackerman, and D. R. Karger. The perfect search engine is not enough: a study of orienteering behavior in directed search. In Proceedings of the SIGCHI conference on Human factors in computing systems, pages 415-422. ACM, 2004.

67. J. Teevan, W. Jones, and B. B. Bederson. Personal information management. Communications of the ACM, 49(1):40-43, 2006.

68. P. Vakkari. Task-based information searching. Annual review of information science and technology, 37(1):413-464, 2003.

69. M. Van Kleek, D. A. Smith, H. S. Packer, J. Skinner, and N. R. Shadbolt. Carpé data: supporting serendipitous data integration in personal information management. In Proceedings of the SIGCHI Conference on Human Factors in Computing Systems, pages 2339-2348. ACM, 2013.

70. M. G. Van Kleek, M. Bernstein, K. Panovich, G. G. Vargas, D. R. Karger, and M. Schraefel. Note to self: examining personal information keeping in a lightweight note-taking tool. In Proceedings of the SIGCHI Conference on Human Factors in Computing Systems, pages 1477-1480. ACM, 2009.

71. E. Von Hippel. Learning from open-source software. MIT Sloan management review, 42(4):82-86, 2001.

72. Y. Wang and A. Kobsa. Privacy-enhancing technologies. Social and Organizational Liabilities in Information Security, pages 203-227, 2006.

73. S. Whittaker. Personal information management: from information consumption to curation. Annual review of information science and technology, 45(1):1-62, 2011.

74. S. Whittaker and J. Hirschberg. The character, value, and management of personal paper archives. ACM Transactions on Computer-Human Interaction (TOCHI), 8(2):150-170, 2001.

75. N. Williams and L. Howard. A sasl and gss-api mechanism for the browserid authentication protocol. 2013.

76. J. Wong and J. I. Hong. Making mashups with marmite: towards end-user programming for the web. In Proc. CHI '07, pages 1435-1444. ACM, 2007.

77. M. Yang, V. Sassone, and K. O'Hara. Appendix 3: practical examples of some anonymisation techniques, pages 80-103. UK Information Commissioner's Office, 2012. 